Cinémas

Revue d'études cinématographiques

Journal of Film Studies

\title{
Document
}

\section{Pierre Francastel. Cours du 2 février 1956. Ambiguiité des figures, pluralité des systèmes}

Volume 19, numéro 2-3, printemps 2009

La filmologie, de nouveau

URI : https://id.erudit.org/iderudit/037558ar

DOI : https://doi.org/10.7202/037558ar

Aller au sommaire du numéro

Éditeur(s)

Cinémas

ISSN

1181-6945 (imprimé)

1705-6500 (numérique)

Découvrir la revue

Citer ce document

(2009). Document / Pierre Francastel. Cours du 2 février 1956. Ambiguïté des

figures, pluralité des systèmes. Cinémas, 19(2-3), 317-326.

https://doi.org/10.7202/037558ar d'utilisation que vous pouvez consulter en ligne.

https://apropos.erudit.org/fr/usagers/politique-dutilisation/ 


\section{Cours du 2 février 1956. Ambiguiité des figures, pluralité des systèmes ${ }^{1}$}

\section{Pierre Francastel}

[p. 2] Lorsqu'on parle de spectacle filmique, il ne s'agit pas seulement d'un phénomène perçu. [On a tendance à oublier ce qui se passe avant la projection or celle-ci n'est qu'un moment.] Avant d'être un phénomène perçu un film est une chose fabriquée et elle n'est pas fabriquée au hasard. Évidemment c'est l'empirisme qui, à l'origine de l'histoire du film, a déterminé la plupart des formules, mais nous en sommes maintenant tout de même à un degré sensiblement plus avancé [s'agissant] de la création filmique elle-même, de son étude, de son observation, d'une réflexion critique sur le phénomène filmique.

Il ne faut jamais oublier qu'avant la projection il y a eu la prise de vue: un film est un produit de la caméra, et n'est pas seulement le déroulement sur un écran du film produit, [il comporte une dimension technique]. C'est, par conséquent, au niveau d'une technique de l'enchaînement des images que se situe, à l'origine, toute espèce de recherche filmologique réfléchie. [...]

Il est indispensable aujourd'hui de partir d'autres bases et ces bases sont nécessairement les conditions dans lesquelles on fabrique la pellicule et pas seulement les conditions dans lesquelles on la projette.

Par conséquent nous avons ici [la] notion de structure du film et également [la] notion de contrat: le spectacle filmique, la préparation de la pellicule ne se font pas tout simplement parce qu'on a trouvé un système qui était préfiguré dans la nature et qui permet de mécaniser le réel, mais parce qu'on a trouvé le moyen de créer un nouveau spectacle humain et ce spectacle implique un certain contrat entre celui qui crée, qui produit la pellicule, le film et celui qui [le] regarde: il y a une sorte de 
connivence, on établit, d'un commun accord, des conventions et ces conventions s'imposent au réalisateur du film dès le premier tour de manivelle. Le simple enregistrement de l'image donne des résultats extrêmement sommaires, ce sont les toutes premières bandes, et encore s'agirait-il de savoir dans quelle mesure elles n'étaient pas orientées par des exemples extrafilmiques. [...]

\section{Des images-signes aux images filmiques}

[p. 6] Ce qu'il faut avant tout éliminer, c'est l'idée, souvent avancée, que l'image filmique apparaît et disparaît simultanément, d'un seul coup, dans son entièreté, que l'image filmique est une chose qui s'impose comme telle: vous êtes dans une salle, devant un écran, vous avez une image; l'image est claire par elle-même, elle est simple, elle est naturelle. Je crois que c'est [la] première illusion qu'il faut dénoncer. La seconde c'est [...] l'illusion qu'il s'agit de l'animation de l'image. En réalité, les deux attitudes se confondent; elles ont comme source commune cette idée que le film consiste à rendre des images du monde extérieur et à les projeter sur un écran: je crois, au contraire, que toute la question va consister à étudier dans quelle mesure il y a fabrication d'une image, une image d'un type particulier, qui n'est ni l'image mentale, ni l'image plastique, ni l'image poétique, littéraire, mais l'image filmique, avec des caractères très spéciaux, très originaux, très déterminés, avec un mécanisme contraignant, qui fait qu'un film apparaît précisément comme doué de réalité, de persuasion, de vérité, ou bien au contraire [apparaît] comme inacceptable, ne rentrant pas dans le domaine des choses vraisemblables. Ce problème se pose d'ailleurs pour tous les arts: c'est le problème du roman: on peut raconter n'importe quelle histoire, à un moment donné on a l'impression que c'est du roman si on arrive à créer un certain degré de vraisemblance. Cette vraisemblance n'est pas du tout liée au degré photographique de réalité, ce n'est pas parce que c'est une copie plus ou moins exacte du réel qu'un roman paraît plus vraisemblable, bien au contraire. De même l'image filmique paraît vraisemblable et naturelle dans la mesure où elle est bien fabriquée, mais elle est fabriquée suivant un certain 
nombre de règles, et non pas seulement extraite en un clin d'œil du réel.

D'ailleurs à ceci il y a des raisons tout à fait évidentes, tout à fait simples, c'est le fameux effet Poudovkine que tout le monde connaît ${ }^{2}$ : une même image mise entre deux images différentes qui la précèdent et qui la suivent, change complètement de signification : le même cliché donnera l'illusion d'un homme qui pleure ou qui sourit suivant la façon dont il aura été encadré par d'autres images. Nous sommes ici en présence d'un fait absolument certain, il n'y a pas une image que l'on anime, il y a un ensemble - l'image filmique - qui est composée d'un certain nombre d'images. C'est toute la notion, d'ailleurs, de découpage et de montage des films qu'on retrouve ici. Il y a un certain nombre d'images qui passent sur l'écran à un rythme déterminé chaque seconde, et, à la suite de la perception d'un certain nombre d'images, il se forme un certain nombre d'autres images qui sont des groupes, c'est-à-dire que dans une succession continue, incessante d'images dont chacune n'est pas absolument caractérisée et différenciée, on détache un certain nombre d'images qui sont les images significatives, que l'on retient. Par conséquent, il faut que nous nous attachions à certaines observations et remarques: il faut distinguer entre la pellicule et l'image, entre l'image qui est sur la pellicule et l'image qui est dans l'esprit du spectateur qui regarde la projection du film. Il y a, naturellement, beaucoup plus d'images sur la pellicule qu'il n'y a d'images intellectuelles; et c'est [pourtant dans] la production de ces images synthétiques qu'est la véritable création du film. Le film, à partir d'un certain nombre d'images enregistrées, crée un certain nombre d'images synthétiques, et c'est dans cette mesure qu'il y a spectacle, et non parce que l'on multiplie un nombre $\mathrm{X}$ d'images indifférentes; le nombre d'images qui passent sur l'écran au rythme de la roue dentée est absolument fixe, mais le nombre d'images que le spectateur perçoit n'est pas déterminé. Il faut quelquefois beaucoup de temps pour préparer et pour produire l'image, quelquefois il suffit de deux ou trois passages d'images pour la modifier et pour faire surgir une nouvelle image. Il y a là toute une liaison qui se joue entre cette 
distinction de l'image qui est sur la pellicule et l'image qui est dans l'esprit du spectateur.

Le film n'a pas d'existence en soi, il est un médium, l'image n'existe que lorsque le film est tourné et présenté. [Ainsi les images de films à la devanture des cinémas ne sont pas directement extraites du film, elles ont été prises pour cette fin] [Lorsque l'on fait passer un film à la moviola [...] on n'a pas l'équivalent de l'image qui apparaît sur un écran quand on projette le film: les conditions filmiques, physiologiques, intellectuelles, affectives ne sont plus remplies: le film n'a sa réalité en tant que telle [p. 10] que dans des conditions très précises de projection cinématographique comprenant l'écran, la salle, etc.]

En outre le motif formel, l'image, n'est pas sur l'écran, sur la pellicule, il est suggéré, il est un signe: nous sommes donc en présence non pas d'une transposition mais de la création d'un système de signes: cette image filmique est un signe parmi les signes. Nous avons un cycle qu'il est impossible de modifier: il faut d'abord qu'une image ait été pensée, filmée, montée, projetée, perçue et qu'elle revienne à la conscience d'un spectateur... [...]

Les appareils pénètrent le réel ${ }^{3}$, c'est une chose absolument incontestable: grâce à la caméra nous avons des possibilités qui n'existaient pas dans les autres générations, de surprendre un certain nombre d'éléments qui sont dans le réel, avant même la projection, qui ne sont pas créés par le film: il ne faut pas en effet prétendre qu'il n'y a d'image que dans la mesure où le film la crée, ce n'est pas vrai: il y a toujours un lien dialectique entre le réel et l'imaginaire. [...]

[p. 11] Le spectateur voit toujours ce qu'on l'amène à voir: les éléments de réalité que le film organise suivant un certain nombre de méthodes, de règles précises, ont été vus pour la première fois par l'auteur du film et son but est d'obtenir que le spectateur les retrouve dans le réel; [...].

On peut imaginer une expérience de ce genre où l'on s'amuserait à associer des images prises par ci par là, sans lien; on ferait passer ensuite au rythme de la présentation filmique une bande ainsi faite: on n'aurait pas un film, mais une image de 


\section{Document}

folie, il n'y aurait pas de cohésion, les images ne se feraient pas: on en aurait tout autant que dans le film ordinaire, mais il n'y aurait pas de film, pas d'images qui passent sous les yeux mais des signes: c'est-à-dire qu'il faut distinguer à la limite entre le signe et l'image, le signe étant le moyen de créer l'image, le signe attirant l'attention sur la valeur de langage du spectacle filmique, mais ne se confondant pas avec l'image filmique, l'image globale. [p. 12] L'image globale [est la] somme d'impressions éprouvées par un spectateur auquel on présente une succession d'images qui en sont les éléments constitutifs.

En bref, le film n'est pas seulement un discours ou un tableau, il est aussi un vocabulaire et une syntaxe: si on prend les lettres d'un mot et qu'on les dispose au hasard, on n'aura pas de mot bien qu'on en ait les éléments: il en est de même pour les images-signes qui constitue l'image filmique à proprement parler. [...]

[Il faut donc maintenant se demander quels sont les ressorts utilisés pour fixer notre attention, extraire du réel des éléments qui ne sont pas indifférents, et pour les ordonner les uns derrière les autres de telle façon qu'à la suite d'une présentation régulière des images-signes, nous voyions apparaître sur notre écran, ou plus exactement dans notre esprit, des images filmiques. Parmi les ressorts de cette création, on peut retenir deux éléments intéressants : l'ambiguïté des figures et la pluralité des systèmes].

\section{[1] Que faut-il entendre par ambiguïté des figures?}

Il y a d'abord cette notion que je viens de rappeler que le film est un [médium], il n'est pas l'image qui a été pensée, vue, par le réalisateur, [ni] l'image qui vient dans l'esprit du spectateur: elle est un intermédiaire, un relais. Il faut [également] tenir compte [du fait] que cette image-relais, signe intermédiaire, est affectée par les considérations que je viens de développer, qu'elle n'est pas simple. J'ai déjà parlé ici de ce que l'on peut appeler les seuils différentiels de la perception ${ }^{4}$ : au moment où l'on regarde un film, on voit des images, le spectacle filmique se forme devant les yeux comme un spectacle continu, sans lacune, dans la mesure où la succession des images répond à certaines règles de la perception physique et physiologique: l'œil, à partir du 
moment où on lui présente un nombre déterminé d'images/ secondes, a la sensation qu'il n'y a pas d'intervalles entre les images, ceci correspondant à un seuil de l'excitation optique qui est un seuil physiologique. Mais précisément, ce n'est pas le même seuil qui permet au spectateur d'avoir la perception claire de l'image mentale: il faut qu'un certain nombre d'images se soient succédé sur l'écran pour que nous percevions, d'une part des présences, d'autre part des changements, la présentation d'une image après l'autre ne suffit pas à donner la sensation du changement, du mouvement, il faut plus d'images physiques que d'images-signes pour former les images filmiques. Nous avons par conséquent une notion de ce que l'on peut appeler les signes perdus, c'est-à-dire que toutes les images qui passent sur l'écran ne sont pas perçues avec la même valeur: il y en a qui servent simplement à assurer la continuité du spectacle, et il y en a d'autres qui jouent un rôle plus grand pour introduire des éléments de modification, pour nous donner le sentiment de ce qui bouge, ce qui se transforme. Autrement dit la présentation du film suit un double tempo et l'un et l'autre sont déterminés par un certain nombre de règles très précises; mais le tempo qui permet d'assurer la sensation d'un continu physique et physiologique de la perception est plus facile à découvrir et à déterminer parce qu'il est l'objet simplement d'expériences physiques et physiologiques, que le tempo qui permet d'assurer le sentiment à la fois de la continuité et du changement des images: ce tempo-là en réalité est réglé par les règles du montage, du découpage; à un moment donné on sait empiriquement qu'il n'est pas besoin de continuer la présentation, on coupe normalement le geste, on interrompt la caméra lorsque l'action est claire: c'est le spectateur qui, par un effort de mémoire, complète l'image; si on s'amusait à aller jusqu'au bout de tous les gestes et toutes les séquences, de tous les mouvements, on aurait un film qui ne marcherait pas, qui traînerait: on n'a pas besoin d'aller jusqu'au bout, à un certain moment, quand on a motivé un acte, l'acte devient clair [...]. Il n'est pas besoin de montrer que la main retombe sur la table pour faire comprendre que je baisse la main. Si on amorce le mouvement à un moment donné, on en anticipe la fin [...]. C'est le rythme filmique [...]. 


\section{Document}

Prenez l'exemple du Ballet mécanique de Léger, vous verrez cette impression de recommencement du geste, qui finit par excé$\operatorname{der}^{5}$ : on a tellement compris que l'intérêt est perdu. Par conséquent, pour retenir l'intérêt, pour créer l'attention, il y a des moments qui ne sont pas indifférents, il y a un rythme du mouvement, de l'enchaînement, non pas cette fois des images-signes, mais des images-film. Ce rythme relève de l'expérience esthétique et non de l'expérience physiologique parce que l'expérience physiologique est toujours satisfaite aussi longtemps qu'il se passe quelque chose régulièrement sur l'écran. [...]

D'où la notion d'ambiguïté des signes filmiques. Un autre élément de cette ambiguiité est dû au fait qu'il y a chevauchement dans ce spectacle d'éléments de perception et d'éléments de mémoire: [...]. Nous ne voyons et nous ne classons une image-signe que dans la mesure où nous nous souvenons de l'image et des images qui l'ont précédée, dans la mesure aussi où nous anticipons sur l'image qui va suivre: nous opérons non seulement sur le perçu immédiat mais à la fois sur ce perçu et sur des éléments d'images-mémoire qui appartiennent au passé individuel de chacun de nous. C'est ce qui fait qu'un film est à la fois un point de rencontre entre tous et une réalité différente pour chacun: chacun lit les images-film d'une manière différente: tout le monde lit l'image-signe de la même manière à peu près, dans un même groupe de civilisation, mais chacun apporte des éléments très différents pour classer, pour reconnaître et pour affecter les structures et les montages qui lui sont suggérés par le réalisateur du film.

Nous avons par conséquent un autre élément qui introduit un caractère d'ambiguïté dans l'image filmique telle que nous la regardons: on ne peut pas comprendre un film, quel qu'il soit, si on ne fait pas appel à notre expérience personnelle, passée, car on ne nous montre que des éléments qui sont caractéristiques et qui n'épuisent jamais toute la définition; on ne comprend Jeux interdits ou Justice est faite 6 que dans la mesure où l'on a une certaine connaissance du milieu humain dans lequel le film a été conçu et dans lequel il a été tourné... [...]

[p. 17] Il y a là une espèce de point d'équilibre extrêmement subtil et difficile à analyser qui repose sur une triple expérience: 
[le continu de la présentation des images-signes, l'expérience collective du groupe humain auquel le film est présenté et la mémoire individuelle de chaque spectateur qui ne coïncide jamais absolument avec la mémoire de son voisin]

Il y a encore toutes sortes de raisons qui nous permettent de prouver ce caractère ambigu des figures de l'image filmique [...], c'est cet effort que nous faisons pour incorporer des images qui n'appartiennent pas à notre souvenir, à notre conscience dans notre esprit: nous découvrons et nous participons parce que nous mettons quelque chose de nous-mêmes dans le spectacle qui nous intéresse: nous ne sommes pas des spectateurs passifs. Par conséquent le film est encore ambigu parce qu'il est constitué à la fois par l'expérience de celui qui l'a fait et par des éléments de l'expérience de chacun de ceux qui le regardent: c'est une fabrication collective, comme d'ailleurs tous les arts plastiques [...].

Autre élément d'ambiguïté: l'image est polysensorielle, elle repose à la fois sur l'expérience acoustique et sur la perception visuelle; et il est incontestable que c'est l'association d'éléments psycho-physiologiques multiples qui donne son évidence: l'image filmique, par cette polysensorialité est encore ambiguë.

On pourrait aussi relever le fait qu'il est rare qu'une image filmique projetée sur un écran ne soit pas un mélange de flou et de net: il est rare que toutes les parties de l'image soient également affectées du même coefficient de netteté; grâce à la profondeur de champ on peut introduire des éléments nets à côté d'éléments flous, c'est là une des ressources du film [...].

En bref il y a toute une technique, toute une série de procédés qui découlent de ce caractère volontairement et nécessairement ambigu de l'image filmique. Créer un champ, le modifier, agencer ensuite des champs — car: ayant dissocié l'image-signe de l'image-filmique, on voit que le film est constitué non seulement d'une succession d'images-signes quasi neutres, quasi indifférentes qui constituent des images filmiques, mais par une succession d'images filmiques agencées les unes avec les autres: c'est à ce moment qu'apparaît le passage du vocabulaire à la syntaxe et au discours - [...] dès lors, avec des images fragmentaires, on peut constituer un film qui est une chose 
éminemment inventée, créée, agencée, organisée: c’est une construction intellectuelle...

Le film est une explication des formes actuelles de l'art contemporain [plus admissible car l'image bouge que dans l'art plastique où l'abstraction propose aussi «un conglomérat d'images qui ont été perçues par l'artiste» mais immobiles elles sont plus difficiles à déchiffrer].

\section{[2] La pluralité des systèmes}

[p. 22] Étant donné cette ambiguïté, on comprend que cette image filmique n'est pas un simple mécanisme mais une «création mentale». [Il est rare que dans un film ne soient présentés qu'un seul espace, un seul temps, une seule causalité:] le plus souvent chaque image participe à différents systèmes [d'où le côté fascinant du cinéma]. Il y a disparité entre les espaces, les temps, la causalité à l'intérieur d'une même image filmique; très souvent les images qui se suivent sont réalisées avec des méthodes qui ne sont pas identiques: c'est un des ressorts de l'attention et de l'intérêt du film; on nous montre, par exemple successivement, une image en gros-plan puis un panoramique, ce sont deux procédés qui mettent en ouvre dans notre esprit des modes de perception très différents, presque opposés, et pourtant nous n'en sommes pas gênés; au contraire, si on ne nous montrait que des gros-plans nous en serions vite fatigués; si on ne nous montrait que des panoramiques, nous n'aurions pas vraiment un film. [...]; cette rupture des systèmes nous oblige à être vigilants, on n'est jamais laissé dans la tranquillité [...]. Il y a toute une série de signes qui n'ont pas de valeur immédiate et qui entrent en combinaison les uns avec les autres d'une façon souple, avec des relais de l'attention pour l'ensemble du film qui est un tout, un bloc et non une succession d'images, mais une construction.

[Cette complexité des systèmes se trouve dans les arts plastiques]: très souvent dans un tableau de l'époque même de la Renaissance, nous voyons associées plusieurs perspectives; on nous montre un personnage au premier plan mais dans le fond il y a un temple, des colonnes, un paysage qui sont vus sous un angle tout à fait différent et cela ne nous gêne pas du tout: notre 
image devient plus riche dans la mesure où l'on fait appel simultanément à des modes de présentation divers.

[...]

\section{Note de la rédaction}

Le texte qui précède est la retranscription par François Albera d'un document déposé en archives (fonds Francastel, Bibliothèque de l'Institut national d'histoire de l'art, à Paris-INHA), qu'il a également annoté et complété. Le texte source est lui-même la transcription d'une intervention orale non révisée par l'auteur. Dans un certain nombre de cas (en particulier en présence de noms propres mal orthographiés ou mal identifiés), les erreurs manifestes dues à la sténographie ont été corrigées. Toutes les autres interventions apparaissent entre crochets.

\section{NOTES}

1. Extraits du cours du 2 février 1956 d'après sa transcription dactylographiée. Publiés avec l'aimable autorisation de M. Philippe Francastel. Voir note de la rédaction ci-dessus.

2. Nom donné au controuvé "effet Kouléchov" jusque dans les années 1950 (Cf. Merleau-Ponty, Hitchcock, etc.). C'est précisément lors de sa venue à l'Institut de filmologie que Poudovkine re-baptisa cette "expérience» qu’il avait relatée ou peutêtre même "inventée " en 1929 dans des conférences à Amsterdam et à Londres, du nom de Kouléchov - lequel n'en avait jamais fait état dans ses écrits jusque dans les années 1970 où il apprend son existence.

3. Expression empruntée à Walter Benjamin dans «L'œuvre d'art à l'époque de la reproduction mécanisée» (p. 52 de l'édition de Maurice Gandillac revue par Rainer Rochlitz, Paris, Allia, 2003).

4. Voir notamment l'article de la Revue internationale de filmologie, $\mathrm{n}^{\circ}$ 5, tome II, $2^{\mathrm{c}}$ année [1949], «Espace et illusion» (1971, p. 171 et suivantes).

5. Il s'agit de la séquence de la lavandière qui gravit un escalier constituée de la répétition d'un même plan en boucle. Francastel a plusieurs fois fait allusion à ce passage. Il est à noter que pour son auteur, Fernand Léger, cette répétition visait précisément à éprouver le degré de résistance des spectateurs à ce type de pratique "aberrante» (Fernand Léger, "Autour du Ballet mécanique» [1927], Fonctions de la peinture, Paris, Gonthier, 1965, p. 138). Gertrude Stein, familière avec ce procédé de répétition dans son écriture, fait allusion dans l'un de ses scénarios ("A Movie» [1920] et «Film — Deux sœurs qui ne sont pas sœurs» [1929], Paris, Plain, 1932) à cette séquence en évoquant une lavandière (rééd. dans Operas and Plays, New York, Station Hill Arts, 1998, p. 395-400) (remerciements à F. Bovier).

6. Justice est faite (réal. : André Cayatte, 1950); Jeux interdits (réal. : René Clément, 1952). 